Article

\title{
Generalizations of Kannan and Reich Fixed Point Theorems, Using Sequentially Convergent Mappings and Subadditive Altering Distance Functions
}

\author{
Alireza Pourmoslemi ${ }^{1, * \mathbb{D}}$, Shayesteh Rezaei ${ }^{2}$, Tahereh Nazari ${ }^{1}$ and Mehdi Salimi ${ }^{3,4, * \mathbb{D}}$ \\ 1 Department of Mathematics, Payame Noor University, P.O. Box 19395-3697, Tehran 43183-14556, Iran; \\ taheree.nazari2222@gmail.com \\ 2 Departement of Mathematics, Aligudarz Branch, Islamic Azad University, Aligudarz 6861885914, Iran; \\ sh.rezaei@iau-aligudarz.ac.ir \\ 3 Center for Dynamics and Institute for Analysis, Department of Mathematics, Technische Universität \\ Dresden, 01069 Dresden, Germany \\ 4 DiGiES and Decisions Lab, University Mediterranea of Reggio Calabria, 89125 Reggio Calabria, Italy \\ * Correspondence: a_pourmoslemy@pnu.ac.ir (A.P.); mehdi.salimi@tu-dresden.de (M.S.)
}

Received: 15 July 2020; Accepted: 17 August 2020; Published: 26 August 2020

\begin{abstract}
In this paper, first, using interpolative Kannan type contractions, a new fixed point theorem has been proved. Then, by applying sequentially convergent mappings and using subadditive altering distance functions, we generalize contractions in complete metric spaces. Finally, we investigate some fixed point theorems which are generalizations of Kannan and Reich fixed points.
\end{abstract}

Keywords: generalized contractions; complete metric space; fixed points; sequentially convergent mappings; subadditive altering distance functions

\section{Introduction and Preliminaries}

Fixed point theory is one of the most popular tools for solving optimization and approximation problems in nonlinear analysis. For the first time, in 1922, Banach introduced a unique fixed point as follows.

Let $(\mathcal{T}, d)$ be a metric space. A mapping $\mathcal{\omega}: \mathcal{T} \rightarrow \mathcal{T}$, is said to be a contraction if there exists $\zeta \in[0,1)$ such that for all $q, s \in \mathcal{T}$

$$
d(\rightsquigarrow(q), \rightsquigarrow(s)) \leq \zeta d(q, s) .
$$

If the metric space $(\mathcal{T}, d)$ is complete then the mapping satisfying (1) has a unique fixed point [1]. In 1968, Kannan [2] and in 1972, Chatterjea [3] studied contractive mappings which give a unique fixed point on a complete metric space. In other words, Kannan stated the following result:

Let $(\mathcal{T}, d)$ be a complete metric space. If a mapping $\omega: \mathcal{T} \rightarrow \mathcal{T}$, satisfies the inequality

$$
d(\rightsquigarrow(q), \rightsquigarrow(s)) \leq \zeta[d(q, \omega(q))+d(s, \rightsquigarrow(s))],
$$

where $\zeta \in\left[0, \frac{1}{2}\right)$ and $q, s \in \mathcal{T}$, then $\omega$ has a unique fixed point [2]. The mappings satisfying (2) are called Kannan type mappings. The significance of the Kannan fixed point theorem appeared in Subrahmanyam paper [4]. He showed that a metric space is complete if and only if every Kannan type mapping has a fixed point. Banach contractions do not have this property; Connell in [5] has given an example of metric space $\mathcal{T}$ that is not complete but every Banach contraction on $\mathcal{T}$ has a fixed point. 
The Banach and Kannan fixed point theorems have been improved by various successful attempts. One such attempt is due to Reich, who proved that if $\vartheta$ is a self-map on a complete metric space $(\mathcal{T}, d)$ that satisfies

$$
d(\vartheta(q), \vartheta(s)) \leq \alpha d(q, \vartheta(q))+\beta d(s, \vartheta(s))+\gamma d(q, s),
$$

where $\alpha, \beta, \gamma \geq 0$ and $\alpha+\beta+\gamma<1$, then $\vartheta$ has a unique fixed point [6]. Note that in (3), $\alpha=\beta=0$ yields Banach's fixed point theorem, while $\alpha=\beta, \gamma=0$ yields Kannan fixed point theorem. The following example shows that this theorem is stronger than Banach and Kannan fixed point theorems.

Example 1 ([6]). Let $\mathcal{T}=[0,1], \vartheta(q)=\frac{q}{3}$ for $0 \leq q<1$ and $\vartheta(1)=\frac{1}{6}$. It is clear that $\vartheta$ is not continuous at $q=1$ and so, it does not satisfy Banach fixed point condition. Now, consider that $d\left(\vartheta(0), \vartheta\left(\frac{1}{3}\right)\right)=\frac{1}{2}\left[d(0, \vartheta(0))+d\left(\frac{1}{3}, \vartheta\left(\frac{1}{3}\right)\right)\right]$. It means that the Kannan fixed point condition cannot be satisfied. On the other hand, if we put $\alpha=\frac{1}{6}, \beta=\frac{1}{9}$ and $\gamma=\frac{1}{3}$, it satisfies (3), and the Reich fixed point is obtained.

Definition 1 ([7]). Let $(\mathcal{T}, d)$ be a metric space. We say, a mapping $v: \mathcal{T} \rightarrow \mathcal{T}$ is sequentially convergent if for each sequence $\left\{q_{n}\right\}$ that $\left\{v\left(q_{n}\right)\right\}$ is convergent then $\left\{q_{n}\right\}$ is also convergent.

In 2011, new generalizations of Kannan fixed point theorem on a complete metric space are investigated as follows:

Theorem 1 ([8]). Let $(\mathcal{T}, d)$ be a complete metric space and $\omega, \vartheta: \mathcal{T} \rightarrow \mathcal{T}$ be mappings such that $\omega$ is continuous, one-to-one, and sequentially convergent. If $0 \leq \zeta<\frac{1}{2}$ and

$$
d(\omega \vartheta(q)), \omega \vartheta(s) \leq \zeta[d(\omega(q), \omega \vartheta(q))+d(\omega(s), \omega \vartheta(s))]
$$

for all $q, s \in \mathcal{T}$, then $\vartheta$ has a unique fixed point.

Singh, Khan and, Fisher [9] in 2012, extended Reich's theorem. Later, In 2016, Malceski and Anevska [10] introduced several extensions of the Kannan fixed point theorem. Hybrid contractions that merge linear and nonlinear contractions in the abstract spaces have been investigated in 2019 [11]. Generalized $(\alpha-\psi)$-Meir-Keeler-Khan mappings in metric spaces [12], fixed points via simulation functions [13], and F-contractions [14,15] are new approaches to the fixed point theory. For further details on state-of-the-art fixed point theorems in metric and normed spaces, see [16-20].

In this paper, Picard iteration is used proving our main results as follows:

Let $(\mathcal{T}, d)$ be a metric space and $\omega: \mathcal{T} \rightarrow \mathcal{T}$ be a mapping. For any $q_{0} \in \mathcal{T}$, the sequence $\left\{q_{n}\right\} \subset \mathcal{T}$ given by

$$
q_{n}=\omega\left(q_{n-1}\right)=\omega^{n}\left(q_{0}\right) \quad n=0,1,2, \ldots
$$

is called the sequence of successive approximations with the initial value $q_{0}$ [21].

Definition 2 ([22]). Let $(\mathcal{T}, d)$ be a metric space. A mapping $\vartheta: \mathcal{T} \rightarrow \mathcal{T}$ is an interpolative Kannan type contraction if there exists a constant $0 \leq \zeta<1$ and $0<\mu<1$ such that

$$
d(\vartheta(q), \vartheta(s)) \leq \zeta[d(q, \vartheta(q))]^{\mu} \cdot[d(s, \vartheta(s))]^{1-\mu},
$$

for all $q, s \in \mathcal{T}$ with $q \neq \vartheta(q)$.

\section{Generalized Kannan and Reich Fixed Point Theorems}

In this section, using interpolative Kannan type contractions, we prove a fixed point theorem in complete metric spaces and then, applying sequentially convergent mappings, subadditive altering distance function and, constants, we generalize Kannan and Reich fixed point theorems. First, it should 
be noted that for ease of reading as well as for avoiding repetition, we set out here some terms that will apply to theorems and corollaries.

Remark 1. In the rest of the paper,

1. let $(\mathcal{T}, d)$ be a complete metric space,

2. let $\omega, \vartheta: \mathcal{T} \rightarrow \mathcal{T}$ be mappings such that $\omega$ is continuous, injective, and sequentially convergent,

3. let $\Psi$ be the class of all nondecreasing continuous functions $\sigma:[0,+\infty) \rightarrow[0,+\infty)$ such that $\sigma^{-1}(0)=0$, unless otherwise stated.

A generalization of Kannan type contraction, applying a continuous, injective, and sequentially convergent map to investigate a unique fixed point is presented in the first theorem.

Theorem 2. If $\zeta \in[0,1), \mu \in(0,1)$, and

$$
d(\omega \vartheta(q), \omega \vartheta(s)) \leq \zeta[d(\omega(q), \omega \vartheta(q))]^{\mu} \cdot[d(\omega(s), \omega \vartheta(s))]^{1-\mu}
$$

for all $q, s \in \mathcal{T}$, then $\vartheta$ has a unique fixed point.

Proof. Suppose that $q_{0} \in \mathcal{T}$ is given and the sequence $\left\{q_{n}\right\}$ be defined as $q_{n+1}=\vartheta\left(q_{n}\right)$ for $n=0,1,2, \ldots$

By taking $q=q_{n}$ and $s=q_{n-1}$ in (4), we get

$$
\begin{aligned}
d\left(\omega\left(q_{n+1}\right), \omega\left(q_{n}\right)\right) & =d\left(\omega \vartheta\left(q_{n}\right), \omega \vartheta\left(q_{n-1}\right)\right) \\
& \leq \zeta\left[d\left(\omega\left(q_{n}\right), \omega \vartheta\left(q_{n}\right)\right)\right]^{\mu} \cdot\left[d\left(\omega\left(q_{n-1}\right), \omega \vartheta\left(q_{n-1}\right)\right)\right]^{1-\mu} \\
& =\zeta\left[d\left(\omega\left(q_{n}\right), \omega\left(q_{n+1}\right)\right)\right]^{\mu} \cdot\left[d\left(\omega\left(q_{n-1}\right), \omega\left(q_{n}\right)\right)\right]^{1-\mu} .
\end{aligned}
$$

Therefore,

$$
\left[d\left(\omega\left(q_{n}\right), \omega\left(q_{n+1}\right)\right)\right]^{1-\mu} \leq \zeta\left[d\left(\omega\left(q_{n-1}\right), \omega\left(q_{n}\right)\right)\right]^{1-\mu} .
$$

We deduce that the sequence $\left\{d\left(\omega\left(q_{n-1}\right), \omega\left(q_{n}\right)\right)\right\}$ is non-increasing and non-negative. Thus, there is a non-negative constant $l$ such that $\lim _{n \rightarrow+\infty} d\left(\omega\left(q_{n-1}\right), \omega\left(q_{n}\right)\right)=l$. From (5) we have

$$
\left[d\left(\omega\left(q_{n}\right), \omega\left(q_{n+1}\right)\right)\right] \leq \zeta\left[d\left(\omega\left(q_{n-1}\right), \omega\left(q_{n}\right)\right)\right] \leq \zeta^{n}\left[d\left(\omega\left(q_{0}\right), \omega\left(q_{1}\right)\right)\right] .
$$

Then for every $n \in \mathbb{N}$ we have,

$$
\begin{aligned}
d\left(\omega\left(q_{n}\right), \omega\left(q_{n+r}\right)\right) & \leq d\left(\omega\left(q_{n}\right), \omega\left(q_{n+1}\right)\right)+\cdots+d\left(\omega\left(q_{n+r-1}\right), \omega\left(q_{n+r}\right)\right) \\
& \left.\leq \zeta^{n}\left[d\left(\omega\left(q_{0}\right), \omega\left(q_{1}\right)\right)\right]+\cdots+\zeta^{n+r-1} d\left(\omega\left(q_{0}\right), \omega\left(q_{1}\right)\right)\right] \\
& \leq \frac{\zeta^{n}}{1-\zeta}\left[d\left(\omega\left(q_{0}\right), \omega\left(q_{1}\right)\right)\right] .
\end{aligned}
$$

Letting $n \rightarrow+\infty$ in the last inequality, we conclude that $\left\{\omega\left(q_{n}\right)\right\}$ is a Cauchy sequence. As the mapping $\omega$ is sequentially convergent, it implies that the sequence $\left\{q_{n}\right\}$ is also convergent, i.e., there exists $p$ such that $\lim _{n \rightarrow+\infty} q_{n}=p$. Since $\omega$ is continuous, $\lim _{n \rightarrow+\infty} \omega\left(q_{n}\right)=\omega(p)$. By taking $q=q_{n}$ and $s=p$ in (4), we find that

$$
d\left(\omega \vartheta\left(q_{n}\right), \omega \vartheta(p)\right) \leq \zeta\left[d\left(\omega\left(q_{n}\right), \omega \vartheta\left(q_{n}\right)\right)\right]^{\mu} \cdot[d(\omega(p), \omega \vartheta(p))]^{1-\mu}
$$

Taking $n \rightarrow+\infty$ in the inequality above, we thus get $d(\omega(p), \omega \vartheta(p))=0$ and since $\omega$ is an injective, we have $\vartheta(p)=p$. 
It remains to prove the uniqueness. Let $\vartheta$ has two distinct fixed points $p, p_{0} \in \mathcal{T}$. Then

$$
\begin{aligned}
d\left(\omega(p), \omega\left(p_{0}\right)\right) & =d\left(\omega \vartheta(p), \omega \vartheta\left(p_{0}\right)\right) \\
& \leq \zeta[d(\omega(p), \omega \vartheta(p))]^{\mu} \cdot\left[d\left(\omega\left(p_{0}\right), \omega \vartheta\left(p_{0}\right)\right)\right]^{1-\mu} \\
& =\zeta[d(\omega(p), \omega(p))]^{\mu} \cdot\left[d\left(\omega\left(p_{0}\right), \omega\left(p_{0}\right)\right)\right]^{1-\mu} \\
& =0 .
\end{aligned}
$$

This shows that $p=p_{0}$.

In the next theorem, a new generalization of Kannan fixed point theorem will be proved.

Theorem 3. If $0 \leq \zeta<\frac{1}{2}, \sigma \in \Psi$ and

$$
\sigma(d(\omega \vartheta(q), \omega \vartheta(s)) \leq \zeta[\sigma(d(\omega(q), \omega \vartheta(q)))+\sigma(d(\omega(s), \omega \vartheta(s)))],
$$

for all $q, s \in \mathcal{T}$, then $\vartheta$ has a unique fixed point.

Proof. Since $\sigma^{-1}(0)=\{0\}$, for every $\varepsilon>0$ we have $\sigma(\varepsilon)>0$. Suppose that $q_{0} \in \mathcal{T}$ is given and the sequence $\left\{q_{n}\right\}$ be defined as $q_{n+1}=\vartheta\left(q_{n}\right)$ for $n=0,1,2, \ldots$.

By taking $q=q_{n-1}$ and $s=q_{n}$ in (6), we get

$$
\begin{aligned}
\sigma\left(d\left(\omega\left(q_{n}\right), \omega\left(q_{n+1}\right)\right)\right) & =\sigma\left(d\left(\omega \vartheta\left(q_{n-1}\right), \omega \vartheta\left(q_{n}\right)\right)\right) \\
& \leq \zeta\left[\sigma\left(d\left(\omega\left(q_{n-1}\right), \omega \vartheta\left(q_{n-1}\right)\right)\right)+\sigma\left(d\left(\omega\left(q_{n}\right), \omega \vartheta\left(q_{n}\right)\right)\right)\right] .
\end{aligned}
$$

So,

$$
\sigma\left(d\left(\omega\left(q_{n}\right), \omega\left(q_{n+1}\right)\right)\right) \leq \frac{\zeta}{1-\zeta} \sigma\left(d\left(\omega\left(q_{n-1}\right), \omega\left(q_{n}\right)\right)\right)
$$

Therefore,

$$
\begin{aligned}
\sigma\left(d\left(\omega\left(q_{n}\right), \omega\left(q_{n+1}\right)\right)\right) & \leq \frac{\zeta}{1-\zeta} \sigma\left(d\left(\omega\left(q_{n-1}\right), \omega\left(q_{n}\right)\right)\right) \\
& \leq\left(\frac{\zeta}{1-\zeta}\right)^{2} \sigma\left(d\left(\omega\left(q_{n-2}\right), \omega\left(q_{n-1}\right)\right)\right) \\
& \leq \cdots \leq\left(\frac{\zeta}{1-\zeta}\right)^{n} \sigma\left(d\left(\omega\left(q_{0}\right), \omega\left(q_{1}\right)\right)\right) .
\end{aligned}
$$

Then, for every $m, n \in \mathbb{N}$ with $m>n$, we have,

$$
\begin{aligned}
\sigma\left(d\left(\omega\left(q_{m}\right), \omega\left(q_{n}\right)\right)\right) & =\sigma\left(d\left(\omega \vartheta\left(q_{m-1}\right), \omega \vartheta\left(q_{n-1}\right)\right)\right) \\
& \leq \zeta\left[\sigma\left(d\left(\omega\left(q_{m-1}\right), \omega\left(q_{m}\right)\right)\right)+\sigma\left(d\left(\omega\left(q_{n-1}\right), \omega\left(q_{n}\right)\right)\right)\right] \\
& \leq \zeta\left[\left(\frac{\zeta}{1-\zeta}\right)^{m-1}+\left(\frac{\zeta}{1-\zeta}\right)^{n-1}\right] \sigma\left(d\left(\omega\left(q_{0}\right), \omega\left(q_{1}\right)\right)\right) .
\end{aligned}
$$

Letting $m, n \rightarrow+\infty$, we get

$$
\lim _{m, n \rightarrow+\infty} \sigma\left(d\left(\omega\left(q_{m}\right), \omega\left(q_{n}\right)\right)\right)=0 .
$$

Since $\sigma \in \Psi, \lim _{m, n \rightarrow+\infty} d\left(\omega\left(q_{m}\right), \omega\left(q_{n}\right)\right)=0$. From this, we conclude that $\omega\left(q_{n}\right)$ is a Cauchy sequence and so, there exists $z \in \mathcal{T}$ such that

$$
\lim _{n \rightarrow+\infty} \omega\left(q_{n}\right)=z
$$


Further, the mapping $\omega: \mathcal{T} \rightarrow \mathcal{T}$ is sequentially convergent and since the sequence $\omega\left(q_{n}\right)$ is convergent, it implies that the sequence $q_{n}$ is also convergent, i.e., there exists $p \in \mathcal{T}$ such that $\lim _{n \rightarrow+\infty} q_{n}=p$. Since $\omega$ is continuous, $\lim _{n \rightarrow+\infty} \mathcal{\omega}\left(q_{n}\right)=\omega(p)$. Thus,

$$
\begin{aligned}
\sigma\left(d\left(\omega \vartheta(p), \omega\left(q_{n+1}\right)\right)\right) & =\sigma\left(d\left(\omega \vartheta(p), \omega \vartheta\left(q_{n}\right)\right)\right) \\
& \leq \zeta\left[\sigma(d(\omega(p), \omega \vartheta(p)))+\sigma\left(d\left(\omega\left(q_{n}\right), \omega \vartheta\left(q_{n}\right)\right)\right)\right] \\
& =\zeta\left[\sigma(d(\omega(p), \omega \vartheta(p)))+\sigma\left(d\left(\omega\left(q_{n}\right), \omega\left(q_{n+1}\right)\right)\right)\right] .
\end{aligned}
$$

Letting $n \rightarrow+\infty$ in (7), we get

$$
\sigma(d(\omega \vartheta(p), \omega(p))) \leq \zeta[\sigma(d(\omega(p), \omega \vartheta(p)))+\sigma(0)] .
$$

Now, from $\sigma^{-1}(0)=0$ and $0<\zeta<\frac{1}{2}$, we conclude that $d(\omega \vartheta(p), \omega(p))=0$. Finally, $\omega$ is an injective map, and thus, $\vartheta(p)=p$.

To prove uniqueness, let $x$ be another fixed point of $\vartheta$. Then by (6), we have

$$
\begin{aligned}
\sigma(d(\omega(p), \omega(x))) & =\sigma(d(\omega \vartheta(p), \omega \vartheta(x))) \\
& \leq \zeta[\sigma(d(\omega(p), \omega \vartheta(p)))+\sigma(d(\omega(x), \omega \vartheta(x)))] \\
& =\zeta[\sigma(d(\omega(p), \omega(p)))+\sigma(d(\omega(x), \omega(x)))]=0 .
\end{aligned}
$$

The last inequality implies that $\sigma(d(\omega(p), \mathfrak{\omega}(x)))=0$, i.e., $\mathscr{\omega}(p)=\mathfrak{\omega}(x)$. This implies that $p=x$.

By taking $\omega(q)=q$ and $\sigma(q)=q$ in Theorem 3, we get the Kannan theorem [2].

Definition 3. A function $\sigma:[0,+\infty) \rightarrow[0,+\infty)$ is called a subadditive altering distance function if

1. $\sigma \in \Psi$,

2. $\sigma(a+b) \leq \sigma(a)+\sigma(b)$ for all $a, b \in[0,+\infty)$.

Example 2. It is easy to check that the functions $\sigma_{1}(a)=a^{\frac{1}{n}}, n \in \mathbb{N}, \sigma_{2}(a)=\log (a+1), a \geq 0$ are such subadditive altering distance functions.

Considering $\sigma$ as a subadditive altering distance function, in the following theorem, we prove another extension of Kannan theorem.

Theorem 4. Let $\sigma$ be a subadditive altering distance function. If $\alpha, \beta, \gamma>0, \alpha+\beta+\gamma<1$, and

$$
\begin{aligned}
\sigma(d(\omega \vartheta(q), \omega \vartheta(s))) & \leq \alpha \sigma(d(\omega(q), \omega \vartheta(q)))+\beta \sigma(d(\omega(s), \omega \vartheta(s))) \\
& +\gamma \sigma(d(\omega(q), \omega(s)))
\end{aligned}
$$

for all $q, s \in \mathcal{T}$, then $\vartheta$ has a unique fixed point.

Proof. Since $\sigma^{-1}(0)=\{0\}$, for every $\varepsilon>0, \sigma(\varepsilon)>0$. Suppose that $q_{0} \in \mathcal{T}$ is given and the sequence $\left\{q_{n}\right\}$ be defined as $q_{n+1}=\vartheta\left(q_{n}\right)$ for $n=0,1,2, \ldots$ By taking $q=q_{n-1}$ and $s=q_{n}$ in (8) we get

$$
\begin{aligned}
\sigma\left(d\left(\omega\left(q_{n}\right), \omega\left(q_{n+1}\right)\right)\right) & =\sigma\left(d\left(\omega \vartheta\left(q_{n-1}\right), \omega \vartheta\left(q_{n}\right)\right)\right) \\
& \leq \alpha \sigma\left(d\left(\omega\left(q_{n-1}\right), \omega \vartheta\left(q_{n-1}\right)\right)\right)+\beta \sigma\left(d\left(\omega\left(q_{n}\right), \omega \vartheta\left(q_{n}\right)\right)\right) \\
& +\gamma \sigma\left(d\left(\omega\left(q_{n-1}\right), \omega\left(q_{n}\right)\right)\right) .
\end{aligned}
$$

Therefore, 


$$
(1-\beta) \sigma\left(d\left(\omega\left(q_{n}\right), \omega\left(q_{n+1}\right)\right)\right) \leq(\alpha+\gamma) \sigma\left(d\left(\rightsquigarrow\left(q_{n-1}\right), \rightsquigarrow\left(q_{n}\right)\right)\right)
$$

Putting $\zeta=\frac{\alpha+\gamma}{1-\beta}$, it follows that

$$
\begin{aligned}
\sigma\left(d\left(\omega\left(q_{n}\right), \omega\left(q_{n+1}\right)\right)\right) & \leq \zeta \sigma\left(d\left(\omega\left(q_{n-1}\right), \omega\left(q_{n}\right)\right)\right) \\
& \leq \zeta^{2} \sigma\left(d\left(\omega\left(q_{n-2}\right), \omega\left(q_{n-1}\right)\right)\right) \\
& \leq \cdots \leq \zeta^{n} \sigma\left(d\left(\omega\left(q_{0}\right), \omega\left(q_{1}\right)\right)\right) .
\end{aligned}
$$

By (9), for all $m, n \in \mathbb{N}$ that $n<m$, we have

$$
\begin{aligned}
\sigma\left(d\left(\omega\left(q_{m}\right), \omega\left(q_{n}\right)\right)\right) & \leq \sigma\left(d\left(\omega\left(q_{m}\right), \omega\left(q_{m-1}\right)\right)+d\left(\omega\left(q_{m-1}\right), \omega\left(q_{m-2}\right)\right)\right. \\
& \left.+\cdots+d\left(\omega\left(q_{n+1}\right), \omega\left(q_{n}\right)\right)\right) \\
& \leq \sigma\left(d\left(\omega\left(q_{m}\right), \omega\left(q_{m-1}\right)\right)\right)+\sigma\left(d\left(\omega\left(q_{m-1}\right), \omega\left(q_{m-2}\right)\right)\right) \\
& +\cdots+\sigma\left(d\left(\omega\left(q_{n+1}\right), \omega\left(q_{n}\right)\right)\right) \\
& \leq\left(\zeta^{m-1}+\zeta^{m-2}+\cdots+\zeta^{n}\right) \sigma\left(d\left(\omega\left(q_{0}\right), \omega\left(q_{1}\right)\right)\right) .
\end{aligned}
$$

So,

$$
\sigma\left(d\left(\omega\left(q_{m}\right), \omega\left(q_{n}\right)\right)\right) \leq \frac{\zeta^{n}}{1-\zeta} \sigma\left(d\left(\omega\left(q_{0}\right), \omega\left(q_{1}\right)\right)\right) .
$$

Letting $m, n \rightarrow+\infty$ in (10), we see that $\left\{\omega\left(q_{n}\right)\right\}$ is a Cauchy sequence in $\mathcal{T}$. Thus, there exists $p_{0} \in \mathcal{T}$ such that

$$
\lim _{n \rightarrow+\infty} \mathscr{\omega}\left(q_{n}\right)=p_{0}
$$

Further, the mapping $\omega: \mathcal{T} \rightarrow \mathcal{T}$ is sequentially convergent. Since the sequence $\mathscr{\omega}\left(q_{n}\right)$ is convergent, it implies that the sequence $q_{n}$ is also convergent, i.e., there exists $p \in \mathcal{T}$ such that $\lim _{n \rightarrow+\infty} q_{n}=p$. Since $\omega$ is continuous, $\lim _{n \rightarrow+\infty} \mathcal{\omega}\left(q_{n}\right)=\mathscr{\omega}(p)$. Thus,

$$
\begin{aligned}
\sigma\left(d\left(\omega \vartheta(p), \omega\left(q_{n+1}\right)\right)\right) & =\sigma\left(d\left(\omega \vartheta(p), \omega \vartheta\left(q_{n}\right)\right)\right) \\
& \leq \alpha \sigma(d(\omega(p), \omega \vartheta(p)))+\beta \sigma\left(d\left(\omega\left(q_{n}\right), \omega \vartheta\left(q_{n}\right)\right)\right) \\
& +\gamma \sigma\left(d\left(\omega(p), \omega\left(q_{n}\right)\right)\right) \\
& =\alpha \sigma(d(\omega(p), \omega \vartheta(p)))+\beta \sigma\left(d\left(\omega\left(q_{n}\right), \omega\left(q_{n+1}\right)\right)\right) \\
& +\gamma \sigma\left(d\left(\omega(p), \omega\left(q_{n}\right)\right)\right) .
\end{aligned}
$$

Now, letting $n \rightarrow+\infty$ in (11), we get

$$
\sigma(d(\omega \vartheta(p), \omega(p))) \leq \alpha \sigma(d(\omega(p), \omega \vartheta(p)))+\beta \sigma(0)+\gamma \sigma(0) .
$$

Since $\sigma^{-1}(0)=0$ and $0<\alpha<1$, we get $d(\omega \vartheta(p), \omega(p))=0$ and thus $\vartheta(p)=p$.

To prove uniqueness, let $x$ be another fixed point of $\vartheta$. Then by (8), we have

$$
\begin{aligned}
\sigma(d(\omega(p), \omega(x))) & =\sigma(d(\omega \vartheta(p), \omega \vartheta(x))) \\
& \leq \alpha \sigma(d(\omega(p), \omega \vartheta(p)))+\beta \sigma(d(\omega(x), \omega \vartheta(x))) \\
& +\gamma \sigma(d(\omega(p), \omega(x))) \\
& =\alpha \sigma(d(\omega(p), \omega(p)))+\beta \sigma(d(\omega(x), \omega(x))) \\
& +\gamma \sigma(d(\omega(p), \omega(x))) \\
& =\gamma \sigma(d(\omega(p), \omega(x))) .
\end{aligned}
$$

Since $0<\gamma<1$, the last inequality implies that $\sigma(d(\omega(p), \mathscr{\omega}(x)))=0$, i.e., $\mathscr{\omega}(p)=\mathscr{\omega}(x)$. Finally, the injectivity of $\omega$ implies $p=x$. 
By taking $\sigma(q)=q$ in Theorem 4, we can conclude the Singh fixed point theorem [9] and by taking $\omega(q)=q$ and $\sigma(q)=q$ in Theorem 4, Reich's fixed point theorem will be obtained [6].

Corollary 1. Let $\sigma$ be a subadditive altering distance function. If $\zeta \in\left[0, \frac{1}{2}\right)$, and

$$
\sigma(d(\omega \vartheta(q), \omega \vartheta(s))) \leq \zeta[\sigma(d(\omega(q), \omega \vartheta(q)))+\sigma(d(\omega(s), \omega \vartheta(s)))]
$$

for all $q, s \in \mathcal{T}$, then $\vartheta$ has a unique fixed point.

In the following example, using Theorem 4, an extension of Kannan theorem is shown.

Example 3. Let $\mathcal{T}=\{0\} \cup\left\{4^{-1}, 5^{-1}, \ldots\right\}$ endowed with the Euclidean metric. Let $\vartheta: \mathcal{T} \rightarrow \mathcal{T}$ defined by

$$
\vartheta(q)= \begin{cases}0 & ; q=0 \\ (n+1)^{-1} & ; q=n^{-1},\end{cases}
$$

for each $n \geq 4$. Obviously the inequality (2) is not held for $\vartheta$ for every $\zeta>0$ and so, we cannot use the Kannan fixed point theorem in this case. Defining $\omega: \mathcal{T} \rightarrow \mathcal{T}$ by

$$
\omega(q)= \begin{cases}0 & ; q=0 \\ \frac{1}{n^{n}} & ; q=n^{-1} .\end{cases}
$$

for $n \geq 4$, follows that $\omega$ is continuous, injective, and sequentially convergent.

Therefore, for all $m, n \in \mathbb{N}, n<m$, we have

$$
\begin{aligned}
\left|\omega \vartheta\left(n^{-1}\right)-\omega \vartheta\left(m^{-1}\right)\right| & =\left|\frac{1}{(n+1)^{n+1}}-\frac{1}{(m+1)^{m+1}}\right|<\frac{1}{(n+1)^{n+1}} \\
& \leq \frac{1}{3}\left(\frac{1}{n^{n}}-\frac{1}{(n+1)^{n+1}}\right) \\
& \leq \frac{1}{3}\left(\frac{1}{n^{n}}-\frac{1}{(n+1)^{n+1}}+\frac{1}{m^{m}}-\frac{1}{(m+1)^{m+1}}\right) \\
& =\frac{1}{3}\left(\left|\omega\left(n^{-1}\right)-\omega \vartheta\left(n^{-1}\right)\right|+\left|\omega\left(m^{-1}\right)-\omega \vartheta\left(m^{-1}\right)\right|\right) .
\end{aligned}
$$

Finally, by Corollary (1), $\vartheta$ has a unique fixed point.

Similar to Theorem 4, the following theorem could be proved. Because of the similarities, we ignore the proof.

Theorem 5. If $\alpha, \beta, \gamma>0, \alpha+\beta+\gamma<1$, and

$$
\begin{aligned}
\sigma(d(\omega \vartheta(q), \omega \vartheta(s)) & \leq \alpha \sigma(d(\omega(q), \omega \vartheta(s)))+\beta \sigma(d(\omega(s), \omega \vartheta(q))) \\
& +\gamma \sigma(d(\omega(q), \omega(s))),
\end{aligned}
$$

for all $q, s \in \mathcal{T}$, then $\vartheta$ has a unique fixed point.

Theorem 6. Let $\sigma$ be a subadditive altering distance function. If $\zeta>0, \mu \geq 0,2 \zeta+\mu<1$, and

$$
\begin{aligned}
\sigma(d(\omega \vartheta(q), \omega \vartheta(s)) & \leq \zeta[\sigma(d(\omega(q), \omega \vartheta(q)))+\sigma(d(\omega(s), \omega \vartheta(s)))] \\
& +\mu \sigma(d(\omega(q), \omega(s))),
\end{aligned}
$$

for all $q, s \in \mathcal{T}$, then $\vartheta$ has a unique fixed point. 
Proof. Suppose that $q_{0} \in \mathcal{T}$ is given and the sequence $\left\{q_{n}\right\}$ be defined as $q_{n+1}=\vartheta\left(q_{n}\right)$ for $n=0,1,2, \ldots$ By taking $q=q_{n-1}$ and $s=q_{n}$ in (13), we get

$$
\begin{aligned}
\sigma\left(d\left(\omega\left(q_{n}\right), \omega\left(q_{n+1}\right)\right)\right) & =\sigma\left(d\left(\omega \vartheta\left(q_{n-1}\right), \omega \vartheta\left(q_{n}\right)\right)\right. \\
& \leq \zeta\left[\sigma\left(d\left(\omega\left(q_{n-1}\right), \omega \vartheta\left(q_{n-1}\right)\right)\right)+\sigma\left(d\left(\omega\left(q_{n}\right), \omega \vartheta\left(q_{n}\right)\right)\right)\right] \\
& +\mu \sigma\left(d\left(\omega\left(q_{n-1}\right), \omega\left(q_{n}\right)\right)\right) .
\end{aligned}
$$

Therefore,

$$
\sigma\left(d\left(\omega\left(q_{n}\right), \omega\left(q_{n+1}\right)\right)\right) \leq \alpha \sigma\left(d\left(\omega\left(q_{n-1}\right), \omega\left(q_{n}\right)\right)\right)
$$

for each $n=0,1,2, \ldots$, and $0<\alpha=\frac{\zeta+\mu}{1-\zeta}<1$.

So,

$$
\begin{aligned}
\sigma\left(d\left(\omega\left(q_{n}\right), \omega\left(q_{n+1}\right)\right)\right) & \leq \alpha \sigma\left(d\left(\rightsquigarrow\left(q_{n-1}\right), \omega\left(q_{n}\right)\right)\right) \\
& \leq \alpha^{2} \sigma\left(d\left(\omega\left(q_{n-2}\right), \omega\left(q_{n-1}\right)\right)\right) \\
& \leq \cdots \leq \alpha^{n} \sigma\left(d\left(\omega\left(q_{0}\right), \omega\left(q_{1}\right)\right)\right) .
\end{aligned}
$$

By (14), for all $m, n \in \mathbb{N}$ with $n<m$, we have

$$
\begin{aligned}
\sigma\left(d\left(\omega\left(q_{m}\right), \omega\left(q_{n}\right)\right)\right) & \leq \sigma\left(d\left(\omega\left(q_{m}\right), \omega\left(q_{m-1}\right)\right)+d\left(\omega\left(q_{m-1}\right), \omega\left(q_{m-2}\right)\right)\right. \\
& \left.+\cdots+d\left(\omega\left(q_{n+1}\right), \omega\left(q_{n}\right)\right)\right) \\
& \leq \sigma\left(d\left(\omega\left(q_{m}\right), \omega\left(q_{m-1}\right)\right)\right)+\sigma\left(d\left(\omega\left(q_{m-1}\right), \omega\left(q_{m-2}\right)\right)\right) \\
& +\cdots+\sigma\left(d\left(\omega\left(q_{n+1}\right), \omega\left(q_{n}\right)\right)\right) \\
& \leq\left(\alpha^{m-1}+\alpha^{m-2}+\cdots+\alpha^{n}\right) \sigma\left(d\left(\omega\left(q_{0}\right), \omega\left(q_{1}\right)\right)\right) .
\end{aligned}
$$

Therefore,

$$
\sigma\left(d\left(\omega\left(q_{m}\right), \omega\left(q_{n}\right)\right)\right) \leq \frac{\alpha^{n}}{1-\alpha} \sigma\left(d\left(\omega\left(q_{0}\right), \omega\left(q_{1}\right)\right)\right) .
$$

This means that $\left\{\mathscr{\omega}\left(q_{n}\right)\right\}$ is a Cauchy sequence. There exists $z \in \mathcal{T}$ such that

$$
\lim _{n \rightarrow+\infty} \omega\left(q_{n}\right)=z
$$

Further, the mapping $\omega: \mathcal{T} \rightarrow \mathcal{T}$ is sequentially convergent. Since the sequence $\mathscr{\omega}\left(q_{n}\right)$ is convergent, it implies that the sequence $q_{n}$ is also convergent, i.e., there exists $p \in \mathcal{T}$ such that $\lim _{n \rightarrow+\infty} q_{n}=p$. Since $\omega$ is continuous, $\lim _{n \rightarrow+\infty} \mathscr{\omega}\left(q_{n}\right)=\mathscr{\omega}(p)$. Thus,

$$
\begin{aligned}
\sigma\left(d\left(\omega \vartheta(p), \omega\left(q_{n+1}\right)\right)\right) & =\sigma\left(d\left(\omega \vartheta(p), \omega \vartheta\left(q_{n}\right)\right)\right) \\
& \leq \zeta\left[\sigma(d(\omega(p), \omega \vartheta(p)))+\sigma\left(d\left(\omega\left(q_{n}\right), \omega \vartheta\left(q_{n}\right)\right)\right)\right] \\
& +\mu \sigma\left(d\left(\omega(p), \omega\left(q_{n}\right)\right)\right) \\
& =\zeta\left[\sigma(d(\omega(p), \omega \vartheta(p)))+\sigma\left(d\left(\omega\left(q_{n}\right), \omega\left(q_{n+1}\right)\right)\right)\right] \\
& +\mu \sigma\left(d\left(\omega(p), \omega\left(q_{n}\right)\right)\right) .
\end{aligned}
$$

Now, letting $n \rightarrow+\infty$ in (15), we get

$$
\sigma(d(\omega \vartheta(p), \omega(p))) \leq \zeta[\sigma(d(\omega(p), \omega \vartheta(p)))+\sigma(0)]+\mu \sigma(0) .
$$

But $d(\omega \vartheta(p), \omega(p))=0$, since $\sigma^{-1}(0)=0$ and $0<\zeta<1$. Thus, $\vartheta(p)=p$.

To prove uniqueness, let $x$ be another fixed point of $\vartheta$. Then by (14), we have 


$$
\begin{aligned}
\sigma(d(\omega(p), \omega(x))) & =\sigma(d(\omega \vartheta(p), \omega \vartheta(x))) \\
& \leq \zeta[\sigma(d(\omega(p), \omega \vartheta(p)))+\sigma(d(\omega(x), \omega \vartheta(x)))] \\
& +\mu \sigma(d(\omega(p), \omega(x))) \\
& =\zeta[\sigma(d(\omega(p), \omega(p)))+\sigma(d(\omega(x), \omega(x)))] \\
& +\mu \sigma(d(\omega(p), \omega(x))) \\
& =\mu \sigma(d(\omega(p), \omega(x))) .
\end{aligned}
$$

Since $0<\mu<1$, the last inequality implies that $\sigma(d(\omega(p), \omega(x)))=0$, i.e., $\omega(p)=\omega(x)$. Finally, the injectivity of $\omega$ implies $p=x$.

By taking $\sigma(q)=q$ in Theorem 6, we can conclude the new extension of Kannan fixed point theorem presented in [10].

Corollary 2. If $0<\zeta<\frac{1}{2}$, and

$$
d(\omega \vartheta(q), \omega \vartheta(s)) \leq \zeta(d(\omega(q), \omega \vartheta(q))+d(\omega(s), \omega \vartheta(s)))
$$

for all $q, s \in \mathcal{T}$, then $\vartheta$ has a unique fixed point.

In the next example, we will show that for a finite set with the Euclidean metric Corollary 2 will be true.

Example 4. Let $\mathcal{T}=\{0\} \cup\left\{1,2^{-1}, 3^{-1}, 4^{-1}, \ldots\right\}$ endowed with the Euclidean metric. Let $\vartheta: \mathcal{T} \rightarrow \mathcal{T}$ defined by

$$
\vartheta(q)= \begin{cases}0 & ; q=0 \\ (n+1)^{-1} & ; q=n^{-1},\end{cases}
$$

for each $n \geq 1$. Obviously the inequality (2) is not held for $\vartheta$, for every $\zeta \in\left[0, \frac{1}{2}\right)$. Defining $\boldsymbol{\omega}: \mathcal{T} \rightarrow \mathcal{T}$ by

$$
\omega(q)= \begin{cases}0 & ; q=0 \\ \frac{1}{\left[e^{2 n}\right]} & ; q=n^{-1} .\end{cases}
$$

for $n \geq 1, \omega$ is continuous, injective, and sequentially convergent. Since $[q][s] \leq[q s]$, we have

$$
\left[e^{2}\right]\left[e^{2 n}\right]=7\left[e^{2 n}\right] \leq\left[e^{2} e^{2 n}\right]=\left[e^{2(n+1)}\right] .
$$

Therefore, for all $m, n \in \mathbb{N}, n<m$

$$
\begin{aligned}
\left|\omega \vartheta\left(n^{-1}\right)-\omega \vartheta\left(m^{-1}\right)\right| & =\left|\frac{1}{\left[e^{2(n+1)}\right]}-\frac{1}{\left[e^{2(m+1)}\right]}\right|<\frac{1}{\left[e^{2(n+1)}\right]} \\
& \leq \frac{1}{6}\left(\frac{1}{\left[e^{2 n}\right]}-\frac{1}{\left[e^{2(n+1)}\right]}\right) \\
& \leq \frac{1}{6}\left(\frac{1}{\left[e^{2 n}\right]}-\frac{1}{\left[e^{2(n+1)}\right]}+\frac{1}{\left[e^{2 m}\right]}-\frac{1}{\left[e^{2(m+1)}\right]}\right) \\
& \leq \frac{1}{6}\left(\left|\omega\left(n^{-1}\right)-\omega \vartheta\left(n^{-1}\right)\right|+\left|\omega\left(m^{-1}\right)-\omega \vartheta\left(m^{-1}\right)\right|\right) .
\end{aligned}
$$

Thus, by (16), $\vartheta$ has a unique fixed point.

Similarly, the following theorem could be solved. 
Theorem 7. If $\zeta>0, \mu \geq 0,2 \zeta+\mu<1$, and

$$
\sigma(d(\omega \vartheta(q), \omega \vartheta(s)) \leq \zeta[\sigma(d(\omega(q), \omega \vartheta(s)))+\sigma(d(\omega(s), \omega \vartheta(q)))]+\mu \sigma(d(\omega(q), \omega(s)))
$$

for all $q, s \in \mathcal{T}$, then $\vartheta$ has a unique fixed point.

\section{Conclusions}

In conclusion, this study provided generalizations of a few well-known theorems such as Kannan, Reich, and Singh fixed point theorems. Our most important tools for generalizing these theorems have been the use of Picard iteration, sequentially convergent maps, and subadditive altering distance functions. Moreover, several examples have been presented to verify the results.

Author Contributions: Supervision, M.S.; Writing—original draft, S.R. and T.N.; Writing-review and editing, A.P. and M.S. All authors have read and agreed to the published version of the manuscript.

Funding: This research received no external funding.

Conflicts of Interest: The authors declare no conflict of interest.

\section{References}

1. Banach, S. Sur les operations dans les ensembles abstraits et leurr application aux equations in tegrales. Fundam. Math. 1922, 3, 133-181. [CrossRef]

2. Kannan, R. Some results on fixed points. Bull. Calcutta Math. Soc. 1968, 60, 71-77.

3. Chatterjea, S.K. Fixed point theorems. C. R. Acad. Bulg. Sci. 1972, 25, 727-730. [CrossRef]

4. Subrahmanyam, P.V. Completeness and fixed points. Mon. Math. 1975, 80, 325-330. [CrossRef]

5. Connell, E.H. Properties of fixed point spaces. Proc. Am. Math. Soc. 1959, 10, 974-979. [CrossRef]

6. Reich, S. Some remarks concerning contraction mappings. Can. Math. Bull. 1971, 14, 121-124. [CrossRef]

7. Branciari, A. A fixed point theorem of Banach-Caccippoli type on a class of generalized metric spaces. Publ. Math. Debr. 2000, 57, 45-48.

8. Moradi, S.; Alimohammadi, D. New extensions of kannan fixed theorem on complete metric and generalized metric spaces. Int. J. Math. Anal. 2011, 5, 2313-2320.

9. Singh, A.; Khan, M.S.; Fisher, B. Some fixed point theorem for certain contractive mappings on metric and generalized metric spaces. Can. Math. Bull. 2012, 16, 69-77. [CrossRef]

10. Malceski, A.; Malceski, S.; Anevska, K.; Malceski, R. New extension of kannan and chatterjea fixed point theorem on complete metric spaces. Math. Comput. Sci. 2016, 17, 1-10. [CrossRef]

11. Kamaleldin, A.; Karapinar, E.; Pitea, A.; Shatanawi, W. Hybrid Contractions on Branciari Type Distance Spaces. Mathematics 2019, 7, 994.

12. Arshad, M.; Alshoraify, S.; Shoaib, A.; Ameer, E. Common fixed points for generalized $(\alpha-\psi)$-Meir-KeelerKhan mappings in metric spaces. J. Anal. 2020. [CrossRef]

13. Karapinar, E. Fixed points results via simulation functions. Filomat 2016, 30, 2343-2350. [CrossRef]

14. Karapinar, E.; Fulga, A.; Agarwal, R.P. A survey: F-contractions with related fixed point results. J. Fixed Point Theory Appl. 2020, 22, 1-58. [CrossRef]

15. Wardowski, D. Fixed points of a new type of contractive mappings in complete metric spaces. Fixed Point Theory Appl. 2012, 1, 94. [CrossRef]

16. Ćirić, L.B. Some recent results in metrical fixed point theory. J. Fixed Point Theory Appl. 2007, 2, $195-120$.

17. Gornicki, J. Fixed point theorem for Kannan type mappings. J. Fixed Point Theory Appl. 2017, 19, $2145-2152$. [CrossRef]

18. Gornicki, J. Various extensions of Kannan's fixed point theorem. J. Fixed Point Theory Appl. 2018, 20, 1045-1057. [CrossRef]

19. Mitrović, Z.D.; Radenović, S.; Reich, S.; Zaslavski, A.J. Iterating nonlinear contractive mappings in Banach spaces. Carpath. J. Math. 2020, 36, 287-294.

20. Puiwong, J.; Satit, S. Banach's fixed point theorem, Ćirić's and Kannan's are equivalent via redefining the metric. Rev. Real Acad. Cienc. Exactas Físicas Nat. Ser. A Matemáticas 2020, 114, 1-8. 
21. Berinde, V. Iterative Approximation of Fixed Points; Springer: Berlin, Germany, 2006.

22. Karapinar, E. Revisiting the Kannan type contractions via interpolation. Adv. Theory Nonlinear Anal. Appl. 2018, 2, 85-87. [CrossRef] 